\title{
INFLUENCE HEUREUSE DES VARIATIONS DU PLAN D'EAU \\ DANS LES APPAREILS D INGUBATION
}

\author{
Par M. E. MUSARI) \\ Ingènieur agricole.
}

Dans un précédent article ( $r$ ) j'ai signalé le gros avantage qu'il y avait, pour empêcher les dépôts de limen sur les ouís de Salmonides en cours d'évolution, à faire varier le niveau de l'eau dans les appareils d'incubation.

L'opération peut se pratiquer, soit une ou deux fois par jour, à la main, soit par adjonction à la sortie d'un siphon qui s'amorce et désamorce auto. matiquement à inlervalles aussi maprochés qu'on le désire.

Le second mode, est préférablr. Il est d'abord plus prailique. Ensuite et surloul il est apparu que les allernances de monlée et baisse de l'eau, non seulement empiohent or dépil sur les arufs, mais ont une influence netle : ur lé díveloppement de l'embryon

Il est remarquable que les alevins provenaul des bacs agencés comme l'a indiqué l'article susvisé sont plus viggourcux et de venue plus régulière que les autres.

Voici commenl nous paraît s'expliquer celte aclion bienfaisante des oscillations relativement fréquentes du plan d'eau.

Dans des appareils d'incubation de système queleonque, pour procéder à une désinfection, il est usuel d'introduire une solution de permanganale de potasse. Il est très facile de voir comment s'opère la diffusion de ce liquide fortement coloré : clle se révèle très souvent irrégulière ; c'est la preuve que les cuffs sont très diversement irrigrués, solon la place gu'ils occupent.

Ivec la stabilité du niveau, les courants qui s’élablissent dans les bars ou augelles sont peu sujets à changements ; de façon constante, certaius reufs sont avantagés, d'autres défavorisés.

II contraire, avec des variations alternatives, l'irrigation est, à chacpuc instant modifiće : tous les aufs se trouvent ainsi soumis en moyenne aux mêmes aclions stimulatrices ou relardatrices du développement embryonnaire.

Quoigu il en soit, d'ailleurs, le fait à retenir, pour les praticiens, est que l'adjonction du siphon automalipue aus appareils d'incubation améliore. de façon sensible, la qualité à l'ó losion des alerins de salmoniculture.

\footnotetext{
(1) Voir Bullelin, n" 91, Janvier ıgat, l, s.í.
} 